\title{
Rat bladder in the early stages of streptozotocin-induced diabetes: adrenergic and cholinergic innervation
}

\author{
J. Lincoln, M.Crockett, A.J. Haven and G. Burnstock \\ Department of Anatomy and Embryology, University College London, London, UK
}

\begin{abstract}
Summary. The adrenergic and cholinergic innervation of the bladder was studied in streptozotocin-diabetic rats. The presence of hypertrophy and distension in the 'diabetic' bladders necessitates care in assessing changes occurring in the nerves, factors which are also relevant to clinical histochemical studies. Biochemical assays of cholinergic enzymes revealed decreased activities per $g$ wet weight tissue. However, the total activities of choline acetyltransferase and acetylcholinesterase per whole bladder were significantly increased after 2 weeks of diabetes with greater changes by 8 weeks. Total dopamine levels per bladder were significantly higher than in control rats in the 2-week but not the 8 -week group of animals; this
\end{abstract}

may indicate an initial increase in adrenergic nerve activity. There was no impairment in the ability of the detrusor muscle to respond to noradrenaline, acetylcholine or to cholinergic nerve stimulation. Shortly after induction of diabetes streptozotocin-treated rats display polyuria. It is proposed that the activity of the bladder is therefore stimulated to allow greater volumes of urine to be passed. The results are discussed in relation to human diabetes mellitus where clinical studies have implicated a neuropathic origin to bladder dysfunction.

Key words: Bladder, diabetic rats, adrenergic nerves, cholinergic nerves, polyuria, neuropathy.
Clinical studies on bladder function in patients suffering from diabetes mellitus have reported that an early indication of dysfunction is increased bladder capacity and size, the incidence of such abnormalities has been reported as being as high as $87 \%$ in a group of juvenileonset diabetic patients [21]. Later stages are associated with atony of the bladder and urinary retention $[16,21$, 29 ], and recent literature reveals that the clinical treatment of these symptoms is still under discussion [19, 32, 39]. Several studies have correlated the presence of abnormalities in the bladder with signs of peripheral neuropathy $[4,5,17]$. In addition, clinical investigations of bladder innervation and function have led to the conclusion that bladder dysfunction is a manifestation of autonomic neuropathy in diabetes mellitus $[3,7,15,20]$. In particular, a defect in the sensory nerves of the bladder has been implicated as the cause of bladder distension in diabetic patients, whilst incomplete bladder emptying occurs as a result of impaired detrusor activity $[5,6,16,36]$. Histochemical studies on diabetic patients [20] and the diabetic Chinese hamster [10] have revealed reduced staining for acetylcholinesterase in the "diabetic' bladder, and consequently it has been suggested that an alteration in the cholinergic innervation of the bladder may be one factor involved in bladder dysfunction.
In the present investigation, bladder dysfunction was studied in the early stages of streptozotocininduced diabetes in rats, an animal model of juvenileonset diabetes. Distension and hypertrophy of the bladder occurred rapidly after the onset of diabetes, and the adrenergic and cholinergic innervation of the bladder was examined using biochemical, pharmacological and histochemical techniques.

\section{Materials and methods}

\section{Induction of diabetes}

Diabetes was induced in adult male Wistar rats (400-450 g) by IP injection of buffered streptozotocin $(65 \mathrm{mg} / \mathrm{kg})$. The onset of diabetes was confirmed by testing the urine for glucose (Clinitest reagent tablets). Animals were maintained for 2- and 8-week periods, food and water being provided ad libitum. Animals of the same initial weight range were used as controls and maintained under identical conditions for similar periods of time. Urinary output was measured in both control and diabetic rats kept in metabolic cages for periods of $24 \mathrm{~h}$. Blood samples for plasma glucose analysis were taken, under ether anaesthesia, from the inferior vena cava immediately before sacrifice. 


\section{Histochemistry}

The entire bladder together with a small portion of the proximal urethra was dissected from 8-week diabetic and control rats. Specimens were emptied of contents, rinsed in Krebs' solution and cut transversely at the mid-point region of the bladder body. Both portions were mounted in cryostat embedding medium and frozen in liquid nitrogen-cooled isopentane. Sections $(10 \mu \mathrm{m})$ were cut using a cryostat at a cabinet temperature of $-20^{\circ} \mathrm{C}$ with a blade cooled by dry ice. Consecutive transverse sections of both the bladder body and the proximal urethra were stained: (a) for catecholamines by the paraformaldehyde fluorescent histochemical method [22] using an incubation period of $1 \mathrm{~h}$; (b) for acetylcholinesterase using a direct staining technique [30]. Incubation with acetylthiocholine iodide was carried out for $1.5 \mathrm{~h}$. Butyrylcholinesterase activity was inhibited by pre-incubation of the sections with tetraisopropylpyrophosphoramide (ISOMPA $10^{-5} \mathrm{~mol} / \mathrm{l}$ ).

\section{Pharmacology}

Strips of detrusor muscle (approximately $10 \mathrm{~mm} \times 3 \mathrm{~mm}$ ) were incubated in modified Krebs' solution [8] at $37^{\circ} \mathrm{C}$ in a $20-\mathrm{ml}$ organ bath bubbled with $\mathrm{O}_{2} / \mathrm{CO}_{2}(95: 5 \%)$. Tissues were allowed to equilibrate for $1 \mathrm{~h}$. Isometric tension was measured using a Grass FT10 force-displacement transducer and was recorded on a Grass Model 7D polygraph (Grass Instruments, Stag Instruments, Henley-on-Thames, UK). An initial load of $0.5-1.0 \mathrm{~g}$ tension was placed on each preparation. Electrical stimulation was carried out at supramaximal voltage using a Grass SD9 stimulator by means of a pair of ring electrodes approximately $3 \mathrm{~mm}$ apart surrounding the muscle strip. Frequencyresponse curves were obtained using stimulations of $10 \mathrm{~s}$ duration with a pulse width of $0.3 \mathrm{~ms}$ over the frequency range $0.5-20 \mathrm{~Hz}$ in the presence and absence of atropine $\left(3.5 \times 10^{-6} \mathrm{~mol} / \mathrm{l}\right)$. Responses of the tissue to acetylcholine were measured over the drug concentration range $10^{-6}-3 \times 10^{-4} \mathrm{~mol} / 1$. The reduction in tissue response to electrical stimulation caused by noradrenaline $\left(10^{-6}-10^{-4} \mathrm{~mol} / \mathrm{l}\right)$ was measured by giving two test stimuli at $10 \mathrm{~Hz}$ (parameters as above) followed by stimulation in the presence of noradrenaline. Recovery of the bladder strips was confirmed by further stimulation after the drug had been washed out. Concentration-response curves to acetylcholine and noradrenaline were both non-cumulative.

\section{Biochemical assays}

Rat bladders were dissected to include the entire body and a small portion of the neck proximal to the entry point of the ureters. Tissue was rinsed in Krebs' solution, blotted dry and immediately frozen and stored in liquid nitrogen.

Noradrenaline and dopamine levels were determined using high performance liquid chromatography with electrochemical detection. The extraction procedure, slightly modified by the addition of $0.1 \mathrm{mmol} / 1 \mathrm{EDTA}$ in the solution used for washing the alumina, was that of Keller et al. [31]. Chromatography was carried out at a flow rate of $1.5 \mathrm{ml} / \mathrm{min}$ using a mobile phase consisting of $0.1 \mathrm{~mol} / 1$ sodium dihydrogen phosphate, $0.1 \mathrm{mmol} / 1$ EDTA, $5 \mathrm{mmol} / 1$ heptane sulphonate ( $\mathrm{pH} 5.0$ ) containing $13 \%(\mathrm{v} / \mathrm{v})$ methanol on a $\mu$-Bondapak $\mathrm{C}-18$ reverse phase column. Detection and quantitation were accomplished with a waxy carbon paste electrode set at a potential of $+0.72 \mathrm{~V}$. Noradrenaline and dopamine levels were corrected for recovery using dihydroxybenzylamine as an internal standard [37].

Whole bladders were homogenised in distilled water (tissue concentration $100 \mathrm{mg} / \mathrm{ml}$ ) using a motor driven glass-glass homogeniser. A spectrophotometric method was used for the estimation of cholinesterase activity with both acetylthiocholine iodide and butyrylthiocholine chloride as substrates [18]. It should be noted that whilst not providing absolute values for butyryl- and acetylcholinesterase activity, the use of both substrates does give an indication of their relative hydrolytic activity. Samples consisted of homogenates $(5 \mathrm{mg} / \mathrm{ml})$ in $0.1 \mathrm{mmol} / 1$ phosphate buffer $(\mathrm{pH} 8.0)$ which had been stored for $<1$ week in liquid nitrogen. Further aliquots were diluted $1: 1$ in $20 \mathrm{mmol} / 1$ EDTA, $2 \%(\mathrm{v} / \mathrm{v})$ Triton X-100 and rehomogenised. These samples were assayed immediately for choline acetyltransferase activity by a radiochemical method [23].

All tissue from diabetic and control rats received identical treatment, and it was confirmed that under the conditions used for the enzyme assays activity was linear with respect to both time and concentration of tissue in the homogenate. It was established that homogenates could be stored for at least 1 week in liquid nitrogen without loss in cholinesterase activity, but choline acetyltransferase had to be assayed immediately after homogenisation.

\section{Source of chemicals}

Acetylcholine chloride, acetylthiocholine iodide, butyrylthiocholine chloride, dopamine hydrochloride, noradrenaline bitartrate (Sigma);

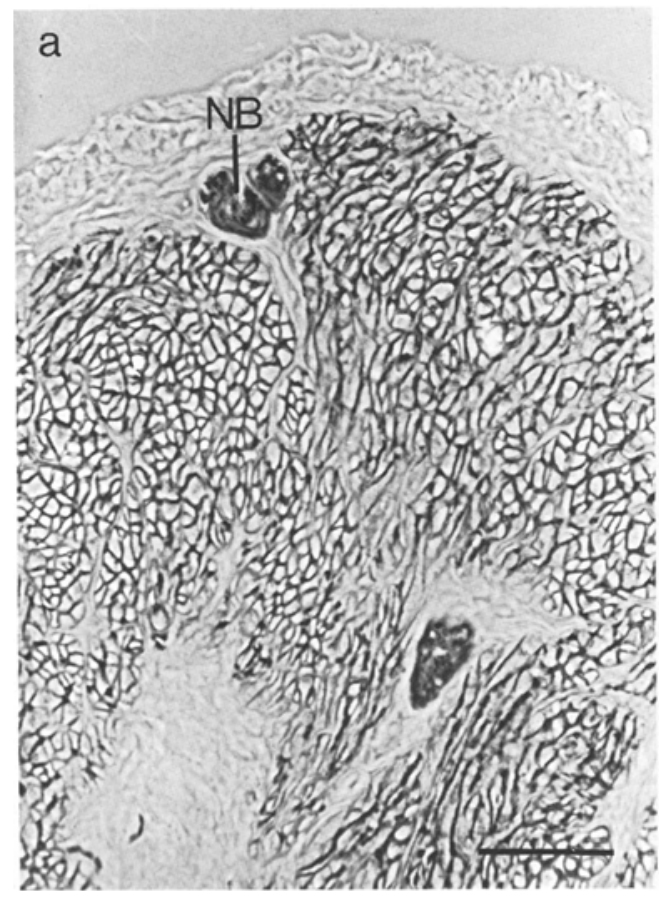

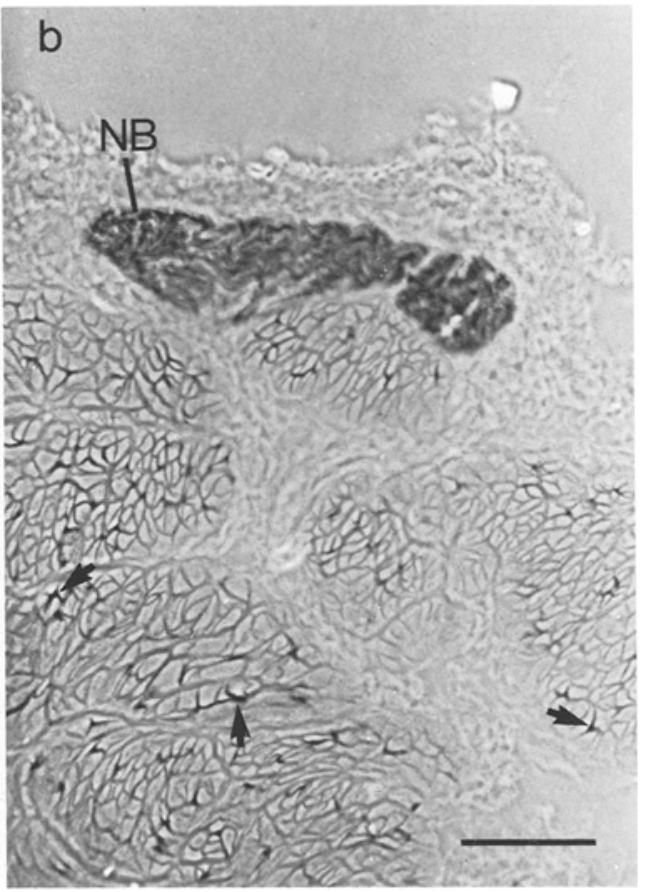

Fig. 1. Acetylcholinesterase staining of $10 \mu \mathrm{m}$ transverse sections of the bladder body examined under phase. (a) Control bladder, (b) bladder from 8-week diabetic rat. Calibration bar $=75 \mu \mathrm{m}$. $\mathrm{NB}=$ nerve bundle. Note the reduced acetylcholinesterase staining, indicated by arrows, around the smooth muscle cells in (b) 

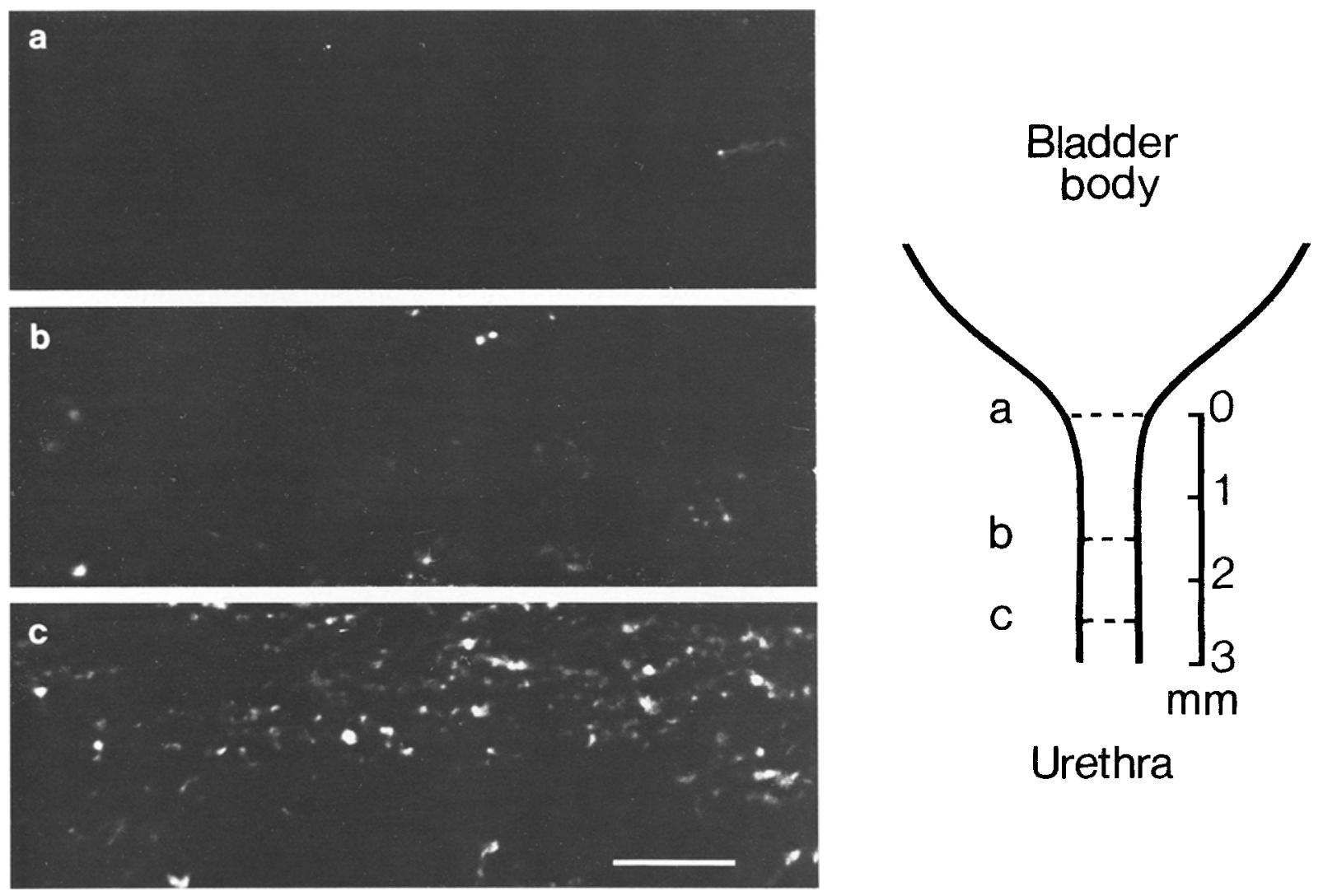

\section{Urethra}

Fig. 2. Fluorescent histochemical staining for catecholamines on $10 \mu \mathrm{m}$ transverse sections of the proximal urethra taken from a control rat. (a) Section adjacent to entry point of the urethra into the bladder body - taken as point zero. (b) Section taken at a distance $1.5 \mathrm{~mm}$ from zeto. (c) Section taken at a distance $2.5 \mathrm{~mm}$ from zero (see diagram). Calibration bar $=30 \mu \mathrm{m}$

heptane sulphonate-sodium salt, dihydroxybenzylamine (Aldrich Chemicals, Gillingham, Dorset, UK), $\mu$-Bondapak C-18 chromatography column (Waters Associates, Northwich, Cheshire, UK); $\left[1-{ }^{14} \mathrm{C}\right]$ acetyl coenzyme A (Amersham International, Amersham, Bucks, UK). All other chemicals used were of analytical grade.

Buffered streptozotocin (NSC-85998) was kindly donated by the Division of Cancer Treatment, National Institutes of Health, Bethesda, Maryland USA.

\section{Statistical methods}

Results are expressed as mean \pm SEM and the data were compared using Student's t-test. A level of probability, $p \leqslant 0.05$ was considered to be significant.

\section{Results}

All diabetic rats showed rapid weight loss, glycosuria and hyperglycaemia. Measurements of 24-h urinary output revealed significant polyuria in diabetic rats $(196 \pm 32.2 \mathrm{ml}$ versus control $17 \pm 9.5 \mathrm{ml} ; n=4$ for each group, $p<0.001)$. On sacrifice, the bladders of diabetic rats were distended, and measurements indicated an increase in total bladder weight. After 8 weeks of diabetes, the weight increase was marked, bladders from diabetic rats being approximately three times heavier than those of control rats $(319 \pm 25.2 \mathrm{mg}, n=17$ versus control: $110 \pm 5.3 \mathrm{mg}, n=13 ; p<0.001)$.

\section{Histochemistry}

The intensity of acetylcholinesterase staining was consistently reduced in the bladder of 8-week diabetic rats when compared with controls, and an example of this is shown in Figure 1. The reduction in staining was found mainly around the muscle fibres in the body of the bladder. The neck region, however, revealed no consistent pattern of change in acetylcholinesterase staining in diabetic tissue.

Catecholamine fluorescence histochemistry revealed sparse adrenergic innervation in the wall of the bladder body which was similar in both diabetic and control rats. In control animals, catecholamine staining changed along the length of the proximal portion of the urethra studied (Fig. 2). Fluorescence intensity decreased as serial sections approached the area where the urethra entered the bladder body. A similar pattern was seen in 8-week diabetic tissue.

\section{Pharmacology}

Detrusor muscle strips responded to exogenous application of acetylcholine by contracting. The atropinesensitive component of the response of the detrusor to electrical stimulation represents stimulation of the cholinergic nerves in the bladder body. The concentration- 

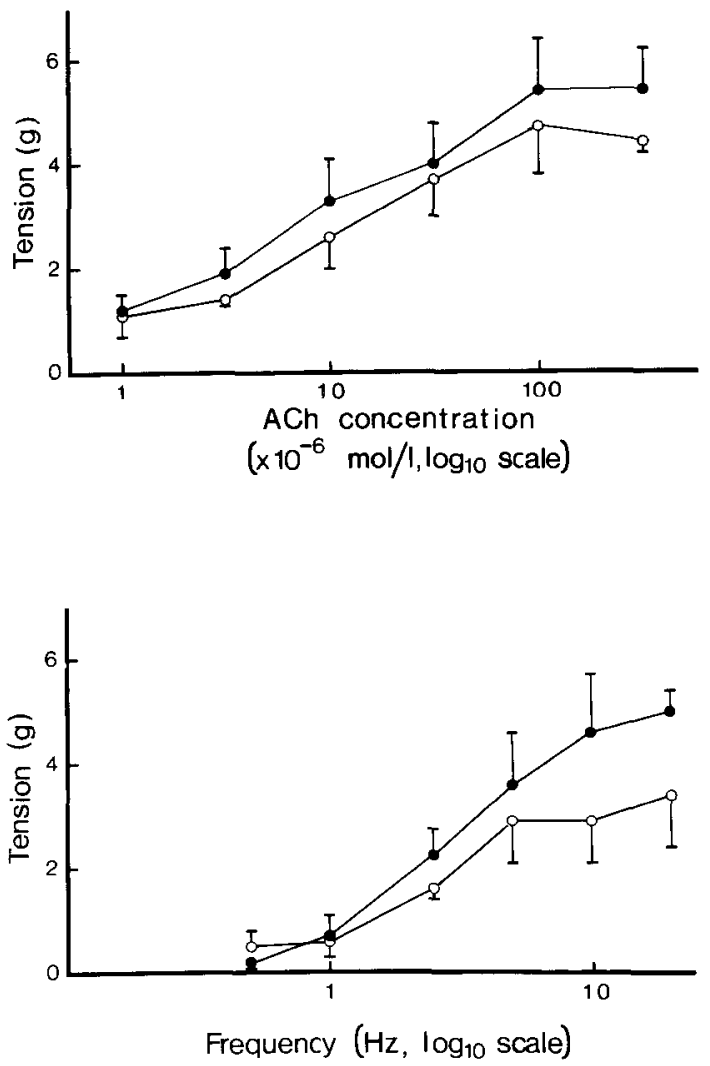

Fig. 3. Effect of 8-week streptozotocin-induced diabetes on the pharmacological response of bladder strips. Upper panel: concentrationresponse curves for acetylcholine (ACh10 $0^{-6}$ to $\left.3 \times 10^{-4} \mathrm{~mol} / 1\right)$. Control: $n=5-8$, diabetic rats: $n=6-7$. Lower panel: frequency-response curves $(0.5-20 \mathrm{~Hz})$ following stimulation of cholinergic nerves, expressed as the atropine-sensitive response to nerve stimulation. Control: $n=5$, diabetic rats: $n=6$. Results are expressed as mean $\pm S E M$. $\mathrm{O}-\mathrm{O}$ control, 2 'diabetic' bladder strips response curves to acetylcholine and the frequency-response curves for cholinergic nerve stimulation are shown in Figure 3. There was no statistically significant difference in either case between control and 8-week diabetic rats. Whilst the sensitivity of the bladder strips to acetylcholine appeared similar in diabetic and control rats, there was a trend towards an increased size of response in the 'diabetic' bladder at all acetylcholine concentrations studied. Similarly, most of the frequencies studied produced a greater response in the 'diabetic' detrusor on stimulation of the cholinergic nerves.

Noradrenaline caused a concentration-dependent reduction in the response of the detrusor to electrical stimulation. There was no difference in either the magnitude of the reduction or the sensitivity to noradrenaline in bladder strips from diabetic rats.

\section{Biochemistry}

Cholinergic enzyme activities have been expressed in terms both of total activity per bladder and activity per $\mathrm{g}$ tissue (Fig.4). Total choline acetyltransferase and acetylcholinesterase activities per bladder were significantly increased in both the 2- and 8-week diabetic animals when compared with controls, with a greater change in the longer time period (2-week diabetic versus 8-week diabetic: $p<0.01$ and $p<0.05$ for choline acetyltransferase and acetylcholinesterase, respectively; Fig.4). The pattern for butyrylcholinesterase, however, was different: whilst an initial significant increase was found after 2 weeks, this appeared to be transient since the 8-week levels were similar in both diabetic and control animals.

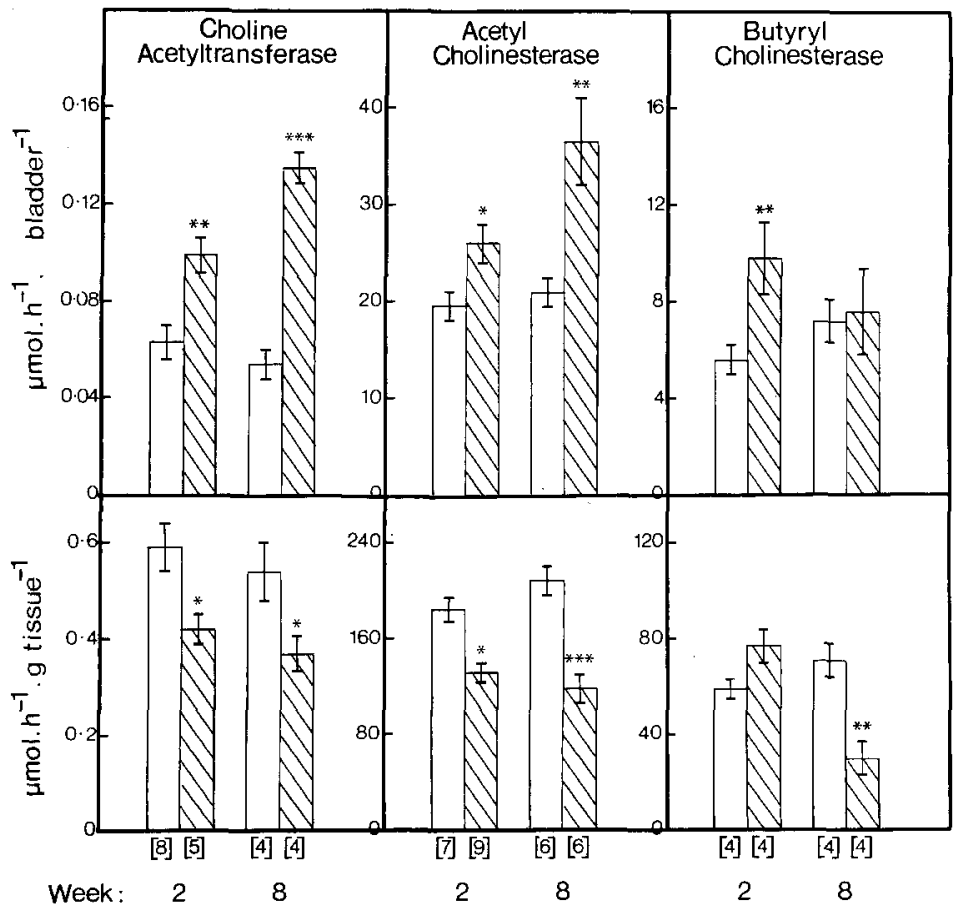

Fig.4. The effect of 2- and 8-week streptozotocin-induced diabetes on choline acetyltransferase, acetylcholinesterase and butyrylcholinesterase activities in the bladder. Upper panels: results expressed as total activity of each enzyme per bladder. Lower panels: results expressed as enzyme activity per $g$ wet weight tissue. The shaded bars represent the values for diabetic and the clear bars, control rats. Results are given as mean $\pm S E M$, with the number of animals used shown in parentheses $\left({ }^{*} p<0.05\right.$, $\left.{ }^{* *} \mathrm{p}<0.01, * * * \mathrm{p}<0.001\right)$ 


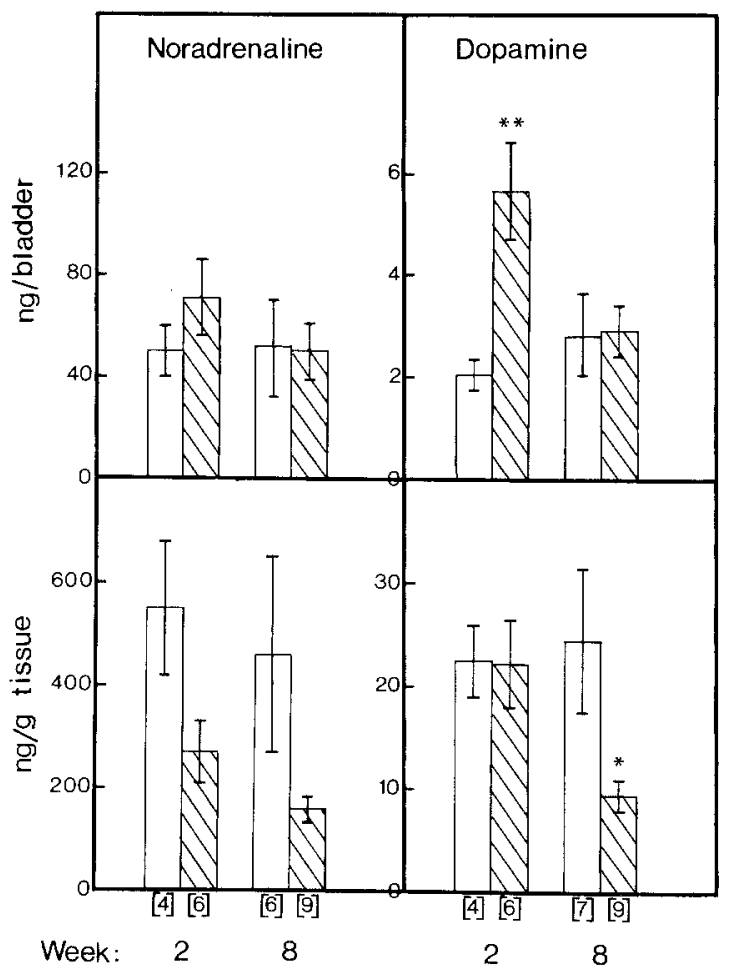

Fig. 5. The effect of 2- and 8-week streptozotocin-induced diabetes on noradrenaline and dopamine levels in the bladder. Upper panels: total content per bladder. Lower panels: content per $g$ wet weight tissue. The shaded bars represent the values for diabetic and the clear bars, control rats. Results are given as mean \pm SEM, the number of animals used is shown in parentheses. $\left({ }^{*} \mathrm{p}<0.05,{ }^{* *} \mathrm{p}<0.01\right)$

When results were expressed per g tissue, statistically significant decreases occurred in both choline acetyltransferase and acetylcholinesterase (Fig. 4). There was no difference between the levels found in bladders at 2 and 8 weeks of diabetes. Butyrylcholinesterase activity was not significantly different from controls 2 weeks after induction of diabetes but was greatly reduced in the 8 -week series.

Noradrenaline and dopamine levels are given in Figure 5. In the 2-week series, there was no significant change in the noradrenaline content per bladder but an approximately threefold increase in dopamine levels was found in the 'diabetic' bladder $(p<0.02$ versus controls). However, following 8 weeks of diabetes, noradrenaline and dopamine levels per bladder were similar to control values. The noradrenaline content per $g$ tissue was decreased in both 2- and 8-week 'diabetic' bladders although the decrease was not statistically significant. Dopamine levels per g tissue were similar to controls after 2 weeks of diabetes, whilst in the 8-week series dopamine levels were significantly reduced.

\section{Discussion}

Cholinergic nerves are partly responsible for contraction of the detrusor muscle facilitating bladder voiding [27]. In the present study, pharmacological experiments revealed a trend towards a greater response in the "diabetic' detrusor to stimulation of cholinergic nerves and exogenous application of acetylcholine. The results were not statistically significant and may reflect different properties in the hypertrophic bladder during contraction; they do not however, reveal any impairment of the ability of the detrusor muscle to contract nor do they suggest a deficit in cholinergic nerve function in the rat bladder in the early stages of streptozotocin-induced diabetes.

A deficit in cholinergic activity in the 'diabetic' bladder has been implicated by reduced histochemical staining for acetylcholinesterase in man and the diabetic Chinese hamster and it has been suggested that this may be responsible for impaired detrusor activity $[10,20]$. A reduction in the density of acetylcholinesterase staining was found in the bladders of the streptozotocin-diabetic rats reported here. However, this may be misleading because in intestinal musculature that is hypertrophic but not denervated, the density of innervation is greatly reduced [25]. Thus, hypertrophy can cause an apparent change in the histochemical staining for acetylcholinesterase without proving a decrease in enzyme activity. This underlines the caution required before accepting histochemical studies alone as evidence of changes in nerve function, especially when distension and hypertrophy occur. Such factors should be taken into consideration both in experimental diabetes and in clinical studies. The present investigation is the first reported study to provide additional biochemical and pharmacological assessment of cholinergic nerve function in the bladder in experimental diabetes.

Choline acetyltransferase activity has frequently been used as a reliable marker of either decreased or increased cholinergic nerve activity. In this study, biochemical measurements of choline acetyltransferase and acetylcholinesterase revealed apparently contradictory results depending on how enzyme activity was expressed. Both methods have been included in the present work because they are relevant to the problems of studying nerve function in a tissue where non-neuronal elements are also undergoing change. The marked increase in total bladder weight invalidates the decrease found in enzyme activities when expressed per g tissue. This argument applies equally to the measurement of noradrenaline and dopamine levels. A similar situation has been described in studies on choline acetyltransferase activity in rat heart and bladder following chemical and surgical treatments which produced both increased and decreased organ weight [12-14]. In these investigations estimations of changes in enzyme activity were based on total activity per organ. Furthermore, it has been shown that a gain in bladder weight or bladder wall stretch does not, per se, cause total choline acetyltransferase activity per bladder to increase [14]. That neither total butyrylcholinesterase activity nor total catecholamine levels per bladder were different from the controls in 8-week diabetic rats further supports the 
conclusion that the increases found in total choline acetyltransferase and acetylcholinesterase activities per bladder represent significant specific changes in cholinergic activity rather than an automatic consequence of increased bladder size. The initial response of the bladder in streptozotocin-diabetic rats therefore involves increased use of the detrusor as demonstrated by hypertrophy with increased total cholinergic enzyme activity per bladder.

Adrenergic innervation of the bladder provides an inhibitory input to the bladder body and an excitatory input to the trigone and smooth muscle of the urethra [27]. Catecholamine fluorescence histochemistry of the normal rat bladder has revealed sparse adrenergic innervation of the detrusor muscle whilst the trigone area and bladder neck have a rich supply of adrenergic fibres [1]. In the present study catecholamine fluorescence was sparse in the bladder wall of both 'diabetic' and control tissue, and pharmacological analysis showed similar concentration-dependent reductions by noradrenaline in the response of 'diabetic' and control detrusor muscle strips to electrical stimulation. The major contribution from adrenergic nerves to noradrenaline and dopamine levels in the bladder arises from the neck region. That fluorescence histochemical staining for catecholamines changes markedly along a small portion of the bladder neck, in both diabetic and control rats, means direct comparison of fluorescence cannot be made in sections from this tissue. Biochemical measurement of noradrenaline and dopamine levels revealed a threefold increase in the total dopamine content per bladder after 2 weeks of diabetes. It has been suggested that increased tissue dopamine levels may be used as an indication of increased adrenergic nerve and tyrosine hydroxylase activity [2]. Should this be the case, the higher dopamine content of 2-week 'diabetic' bladders would imply increased adrenergic activity, resulting in greater contraction of the bladder neck. At present this can only be speculation since SIF cells, which contain catecholamines including dopamine, have been shown to be present in the rat bladder [1]. After 8 weeks there was no difference in the total noradrenaline and dopamine levels of the bladder.

The presence of hypertrophy, distension and increased bladder size in diabetic rats was noted in the present study. Since such features have also been found in non-diabetic rats with increased urinary output [26], it is postulated these changes occur as a result of polyuria. The results reported here show increased cholinergic nerve activity which, in conjunction with hypertrophy, suggest that the activity of the detrusor muscle has been stimulated shortly after induction of diabetes. The initial sequence of events in streptozotocin-diabetic rats therefore appears to involve polyuria; to enable increased volumes of urine to be passed, the bladder distends and increased cholinergic nerve activity stimulates contraction of the detrusor to raise the frequency of micturition which results in hypertrophy of the de- trusor. It can be speculated that before the bladder distends sufficiently to compensate for increased output, adrenergic activity is stimulated in the bladder neck to maintain closure against higher vesicle pressure.

In several clinical studies, it has been suggested that a defect in the sensory innervation of the bladder is the cause of bladder distension and that this may be one of the earliest manifestations of autonomic neuropathy in diabetes $[6,16,36]$. These conclusions have mainly been drawn from the simultaneous detection of increased capacity and diminished bladder sensation to filling in diabetic patients. The present study does not exclude the possibility of damage to sensory nerves occurring shortly after induction of diabetes. It is interesting, however, that chronic overdistension on its own, in the absence of diabetes, can cause decreased sensation resulting in enlarged bladders [24]. Furthermore, functional and ultrastructural changes occur in human and animal detrusor muscles following even single episodes of overdistension [33-35]. The rapid nature of the changes reported here make it apparent that even in newly diagnosed diabetic patients the bladder may require clinical attention particularly with regard to the avoidance of overdistension. The latter stages of impaired detrusor activity and urinary retention present significant problems in clinical treatment. Pharmacological manipulation has been largely ineffective [32], intermittent selfcatheterisation has been recommended [32, 38], and it has been suggested that bladder neck surgery may be required [19].

Longer term studies are required to determine whether chronic hypertrophy, distension and increased urinary output may make the bladder particularly susceptible to autonomic nerve damage resulting in impaired detrusor function and incomplete bladder emptying. For a complete study of nerve function in the 'diabetic' bladder it is also necessary to include the nonadrenergic, non-cholinergic contribution to detrusor activity $[9,11,28]$. Future studies will be extended to longer duration of diabetes and include examination of the pelvic plexus for the abnormalities that have been shown to occur in the diabetic Chinese hamster after 7 months [10]. These abnormalities need to be correlated with detrusor function assessed by biochemical, microscopical and pharmacological analysis in order to determine the significance of neuropathic changes in the autonomic nerves to the latter stages of urinary retention and bladder dysfunction in diabetes mellitus.

Acknowledgements. We wish to thank the Department of Chemical Pathology, University College Hospital for the analysis of plasma glucose samples. We are grateful to J. Bokor both for technical assistance and helpful discussion concerning the manuscript. G. Christaki and A. Thompson provided valuable technical assistance. This work was supported by grants from the British Diabetic Association and the Wellcome Foundation.

\section{References}

1. Alm P, Elmer M (1975) Adrenergic and cholinergic innervation of the rat urinary bladder. Acta Physiol Scand 94: 36-45 
2. Almgren O, Carlsson A, Snider S (1979) Tissue dopamine levels as an indicator of tyrosine hydroxylase activity. In: Imbo J-L, Schwartz J (eds) Advances in the biosciences, Vol 20. Peripheral dopaminergic receptors. Pergamon, Oxford, pp $29-40$

3. Andersen JT, Bradley WE (1976) Abnormalities of bladder innervation in diabetes mellitus. Urology $7: 442-448$

4. Bartley O, Brolin I, Fagerberg SE, Wilhelmsen L (1966) Neurogenic disorders of the bladder in diabetes mellitus: a clinicalroentgenologic investigation. Acta Med Scand 180: 187-199

5. Bradley WE (1980) Diagnosis of urinary bladder dysfunction in diabetes mellitus. Ann Intern Med 92: 323-326

6. Buck AC, McRae CU, Reed PI, Chisholm GD (1974) The diabetic bladder. Proc R Soc Med 67:81-83

7. Buck AC, Reed PI, Siddiq YK, Chisholm GD, Fraser TR (1976) Bladder dysfunction and neuropathy in diabetes. Diabetologia 12: $251-258$

8. Bulbring $\mathrm{E}$ (1953) Measurements of oxygen consumption in smooth muscle. J Physiol 122: 111-134

9. Burnstock G, Cocks T, Crowe R, Kasakov L (1978) Purinergic innervation of the guinea-pig urinary bladder. Br J Pharmacol 63: 125-138

10. Dail W, Evan A, Gerritsen G, Dulin W (1977) Abnormalities in pelvic visceral nerves: $A$ basis for neurogenic bladder in the diabetic Chinese hamster. Invest Urol 15: 161-166

11. Downie JW (1981) The autonomic pharmacology of the urinary bladder and urethra: a neglected area. Trends Pharmacol Sci 2: 163-165

12. Ekström J (1975) Choline acetyltransferase activity in the rat's heart and urinary bladder after chemical sympathectomy. Acta Pharmacol Toxicol 36: 284-288

13. Ekström J (1978) Fall in choline acetyltransferase activity in the ventricles of the rat heart after treatment with a ganglion blocking drug. Acta Physiol Scand 102: 116-119

14. Ekström J (1981) Increase in choline acetyltransferase activity in surgically isolated postganglionic parasympathetic neurones of the urinary bladder of adult rats. Acta Physiol Scand 111: 81-86

15. Ellenberg M (1966) Diabetic neurogenic vesical dysfunction. Arch Intern Med 117: 348-354

16. Ellenberg M (1980) Development of urinary bladder dysfunction in diabetes mellitus. Ann Intern Med 92:321-323

17. Ellenberg M, Weber $H$ (1967) The incipient asymptomatic diabetic bladder. Diabetes 16:331-335

18. Ellman GL, Courtney KD, Andres V, Featherstone RH (1961) A new and rapid colorimetric determination of acetylcholinesterase activity. Biochem Pharmacol 7:88-95

19. Ewing DJ, Clarke, BF (1982) Diagnosis and management of diabetic autonomic neuropathy. Br Med J 285: 916-918

20. Faerman I, Glocer L, Celener D, Jadzinsky M, Fox D, Maler M, Alvarez E (1973) Autonomic nervous system and diabetes: Histological and histochemical study of the autonomic nerve fibres of the urinary bladder in diabetic patients. Diabetes 22: 225-237

21. Faerman I, Maler M, Jadzinsky M, Alvarez E, Fox D, Zilbervar GJ, Cibeira JB, Colinas R (1971) Asymptomatic neurogenic bladder in juvenile diabetics. Diabetologia 7: 168-172
22. Falck B, Hillarp NA, Thieme G, Torp A (1962) Fluorescence of catecholamines and related compounds condensed with formaldehyde. J Histochem Cytochem 10: 348-354

23. Fonnum $F$ (1975) A rapid radiochemical method for the determination of choline acetyltransferase. J Neurochem 24: 407-409

24. Frimodt-Moller C (1980) Diabetic cystopathy: Epidemiology and related disorders. Ann Intern Med 92: 318-321

25. Gabella $G$ (1979) Hypertrophic smooth muscle III. Increase in number and size of gap junctions. Cell Tissue Res 201: 263-276

26. Goss RJ, Liang MB, Weisholtz SJ, Peltzer TJ (1973) The physiological basis of urinary bladder hypertrophy. Proc Soc Exp Biol Med 142: 1332-1335

27. de Groat WC, Booth AM (1980) Physiology of the urinary bladder and urethra. Ann Intern Med 92: 312-315

28. Hökfelt T, Schultzberg M, Elde R, Nilsson G, Terenius L, Said S, Goldstein M (1978) Peptide neurons in peripheral tissues including the urinary tract: immunohistochemical studies. Acta Pharmacol Toxicol 43 (II): $79-89$

29. Kahan M, Goldberg PD, Mandel EE (1970) Neurogenic vesical dysfunction and diabetes mellitus. NY State J Med 2: 2448-2455

30. Karnovsky MJ, Roots L (1964) A 'direct colouring' method for cholinesterases. J Histochem Cytochem 12: 219-221

31. Keller R, Oke A, Mefford I, Adams RN (1976) Liquid chromatographic analysis of catecholamines: routine assay for regional brain mapping. Life Sci 19: 995-1004

32. Kirby R, Billings P, Vordermark J, Pitfield J, Milroy E, TurnerWarwick RT (1982) Diagnosis and management of diabetic autonomic neuropathy. Br Med J 285: 1353

33. Lloyd-Davies RW, Clark AE, Prout AE, Shuttleworth KED, Tighe JR (1970) The effects of stretching the rabbit bladder. Invest Urol $8: 145-152$

34. Lloyd-Davies RW, Hinman F Jr. (1970) Structural alterations and functional changes after overdistension of the rabbit bladder. Surg Forum 21: 542-543

35. Mayo ME, Lloyd-Davies RW, Shuttleworth KED, Tighe JR (1973) The damaged human detrusor: functional and electron miscoscopic changes in disease. Br J Urol 45: 116-125

36. Motzkin D (1968) The significance of deficient bladder sensation. J Urol 100: 445-450

37. Moyer TP, Jiang N-S (1978) Optimized isocratic conditions for analysis of catecholamines by high-performance reversed-phase paired-ion chromatography with amperometric detection. J Chromatogr 153: 365-372

38. Stanton SL (1982) Diagnosis and management of diabetic autonomic neuropathy. Br Med J 285: 1353

Received: 22 November 1982

and in revised form: 5 September 1983

Professor G. Burnstock

Department of Anatomy and Embryology

University College London

Gower Street

London WC1E 6BT, UK 